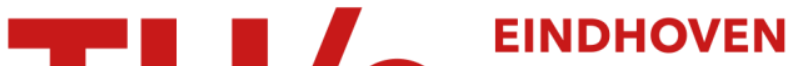 UNIVERSITY OF TECHNOLOGY
}

\section{On the application of uncertainty and sensitivity analysis with abstract building performance simulation tools}

\section{Citation for published version (APA):}

Struck, C., Hensen, J. L. M., \& Kotek, P. (2009). On the application of uncertainty and sensitivity analysis with abstract building performance simulation tools. Journal of Building Physics, 33(1), 5-27.

https://doi.org/10.1177/1744259109103345

DOI:

10.1177/1744259109103345

Document status and date:

Published: 01/01/2009

\section{Document Version:}

Accepted manuscript including changes made at the peer-review stage

\section{Please check the document version of this publication:}

- A submitted manuscript is the version of the article upon submission and before peer-review. There can be important differences between the submitted version and the official published version of record. People interested in the research are advised to contact the author for the final version of the publication, or visit the $\mathrm{DOI}$ to the publisher's website.

- The final author version and the galley proof are versions of the publication after peer review.

- The final published version features the final layout of the paper including the volume, issue and page numbers.

Link to publication

\section{General rights}

Copyright and moral rights for the publications made accessible in the public portal are retained by the authors and/or other copyright owners and it is a condition of accessing publications that users recognise and abide by the legal requirements associated with these rights.

- Users may download and print one copy of any publication from the public portal for the purpose of private study or research.

- You may not further distribute the material or use it for any profit-making activity or commercial gain

- You may freely distribute the URL identifying the publication in the public portal.

If the publication is distributed under the terms of Article 25fa of the Dutch Copyright Act, indicated by the "Taverne" license above, please follow below link for the End User Agreement:

www.tue.nl/taverne

Take down policy

If you believe that this document breaches copyright please contact us at:

openaccess@tue.nl

providing details and we will investigate your claim. 
Struck, C., Hensen, J., \& Kotek, P. (2009).

On the application of uncertainty and sensitivity analysis with abstract building performance simulation tools, Journal of Building Physics, 33 (1), pp. 5-27

\title{
On the Application of Uncertainty and Sensitivity Analysis with Abstract Building Performance Simulation Tools
}

Christian Struck, ${ }^{1, *}$ Jan Hensen ${ }^{1}$ and Petr Kotek ${ }^{2}$

\author{
${ }^{1}$ Unit Building Physics and Systems, Technische Universiteit Eindhoven, \\ Den Dolech 2, Eindhoven 5600MB , Netherlands \\ 2 Department of Civil Engineering, CVUT Technical University of Prague, \\ Czech Republic
}

\begin{abstract}
Building Performance Simulation (BPS) is a powerful measure to educate the building design process. However, its use in practice is too large extents limited to the detailed design stage dedicated to the code compliance analysis of worked out design proposals. BPS is not much used to support the conceptual design stage. To date BPS - tools are regarded as pure analysis tools, which do not provide design information.

It is hypothesized that, when integrating uncertainty analysis techniques to existing BPS tools, following an incremental research approach, valuable design information can be provided.

The paper gives an update on the process to extend the capabilities of a tool specifically developed to support the conceptual design stage about uncertainty assessment. The resulting prototype should be capable of providing information about the variation of specific building performance metrics (simulation output) based on the propagated uncertainties of the building specification (simulation input). The results also indicate the degree of impact, sensitivity, of the building specification parameters on the variation of the performance metrics as a result of their uncertainty.

This paper describes an approach to add extra-capabilities to legacy simulation tools and presents a comparison of uncertainties and sensitivities calculated with one detailed design analysis tool, IES, and one conceptual design analysis tool, LEA. The main focus was herby the representation of material properties and their impact on the performance uncertainty of the building concept.
\end{abstract}

KEYWORDS: Concept design, Uncertainty analysis, Sensitivity analysis, Specification

Uncertainty, Material properties.

\section{INTRODUCTION}

Although practitioners assess the use of Building Performance Simulation (BPS) tools for conceptual design to have the potential to add value too, and to educate the design process, their use is limited. It is argued that the mismatch between the tool capabilities and the user's needs is one of the reasons explaining this phenomenon (Hopfe et al. 2006). Considering the conceptual design stage (CDS) one needs to be aware of its characteristics and their impact on the use of BPS - tools.

The detailed design stage (DDS) is characterized by a limited number of design concepts with extensive parametric detail, which facilitates the use of detailed design analysis (DDA) - tools. The CDS is characterized by a large number of design concepts with none to little parametric detail. By applying DDA - tools, the limited amount of design information available requires assumptions to fill the gaps.

*Author to whom correspondence should be addressed.

E-mail: C.Struck@tue.nl 
Different strategies are deployed to develop concept design analysis (CDA) - tools. One strategy is to abstract the interface by reducing the number of parameters required to define the building and system model. Another strategy is to use simplified physical models. Both strategies when applied reduce the input detail, to describe the building and its use, to the crucial parameters governing energy demand and/or thermal comfort. One example is restricting the definition of more than one or two thermal zones, which traditionally represent spaces or groups of spaces within one building. Another simplification is the definition of the buildings thermal response by defining the building mass directly as number or by options as high and low (Urban and Glicksman 2006; Vabi 2006). Furthermore, it has been observed that CDA - tools frequently use fixed values to represent the surface heat transfer on building elements.

A review of six BPS - tools, advertised as being of use from concept to detailed design, revealed that only two out of six, Energy 10 and h.e.n.k., come close to serving the needs of practitioners during the CDS (Hopfe et al. 2005). Those tools represent analysis tools that allow the analysis of one design proposal at the time. The tool user is left to make interpretations of the numbers representing the tool output and to convert the analysis results to design information. Whilst one simulation might be sufficient for code compliance analysis, a greater number of simulations is required to perform parametric uncertainty and sensitivity studies.

Individual parameter sensitivity studies form one integral part of the concept evaluation phase in design practice. Introducing a capability to conduct building performance sensitivity studies the practitioner is supported with information about the deviation (uncertainty) of selected performance metrics. One example is the assessment of energy demand and thermal comfort variations due to uncertainties in the building specification. Additionally, information indicating sensitivities of the building specification parameters as a result of their impact on the performance metrics can be derived. By integrating sensitivity analysis capabilities, the search space for potential concepts is expected to be explored more thoroughly and structured. The underlying idea is to support design practitioners with an extra measure to better assess the risk of the design failing to meet the design requirements. Therefore, it is proposed to propagate the concepts inherent uncertainties trough a simulation model and establish metric specific performance ranges. Later, the range can be explored by isolating the impact of parameters or subsystems on the range of its performance. It is expected that the sensitivity analysis provides the practitioner with information about which parameters or subsystems to attend to, to reduce the performance uncertainty. This is of particular interest if the performance metric of concern does not comply with the posed performance requirements.

The uncertainties and sensitivities of four performance metrics were calculated, with two tools addressing the impact of uncertainties in material properties for a CDA and DDA - tool. A tool performance comparison has been carried out. The four performance metrics compared are: annual demand for heating and cooling as well as peak heating and cooling loads.

\section{UNCERTAINTY ANALYSIS IN BUILDING PERFORMANCE SIMULATION}

De Wit in (2001) identified four potential sources of uncertainties in BPS. He differentiated between numerical -, scenario-, specification-, and modeling uncertainties. The latter two, specification and modeling uncertainties, addressing the description of the building system, its properties and modeling abstraction level are addressed here.

The aim of earlier efforts to introduce uncertainty analysis techniques to BPS was to more realistically model the real on-site performance of buildings by using data that represent material performance variation factors as aging, moisture content and temperature variations (Struck and Hensen 2006). Others have reported the use of uncertainty analysis for supporting the optimization process of low energy dwellings (Verbeeck and Hens 2007). Here, uncertainty analysis is used to provide design information, which is by nature of the design stage, far from realistic but provides a strategy to systematically explore the performance of concept variations and their sensitivities. 
Two different types of analysis are reported in literature; global and local uncertainty analysis (Lomas and Eppel 1992). Global uncertainty analysis provides a measure of uncertainty by addressing the entire solution space, changing all parameters simultaneously across their full range, whilst local uncertainty analysis, Method of Morris, is used to identify the individual impact of selected input parameters on the predicted performance indicator.

This study considers global uncertainties and sensitivities. The output uncertainty gives the range and probability density of the performance metrics for specific confidence intervals.

The sensitivities are derived from the same output data, based on an input sample matrix that uses different parametric values for each sample. Individual parameter sensitivities, which can be derived from changing only one specification parameter at the time in each sample, are not considered.

\section{METHODOLOGY}

A comparative study was conducted between two uncertainty analysis prototypes. The first prototype, used as reference, is based on a DDA - tool. The second prototype, under investigation, was built around a CDA -tool. Based on the software review the successor of h.e.n.k., LEA prerelease 0.9.3, was chosen for representing the category of CDA - tools. IES, an industry strength and extensively used DDA - tool was chosen to represent the category of detailed design analysis tools. As LEA is specifically designed to support practitioners with estimations of the instantaneous peak heat/ cooling load and annual energy demand for heating/cooling the analysis had to be limited to those four parameters.

\section{Tool description}

The BPS - tools used in this study are commercial programmes for which the underlying code is not distributed under General Public license. However for both tools, IES VE v.5.5.1 and LEA prerelease 0.9.1, a user manual is available from which information of the calculation models can be drawn.

Apachesim the calculation model for the dynamic simulation within IES uses a method which is similar to the Crank-Nicholson discretisation scheme called "hopscotch". This discretisation scheme applies explicit and implicit time-stepping to alternate nodes of the construction. The advantages of this method are a high level of accuracy combined with very efficient computation. To model the thermal response of the structure the admittance method is implemented (Crawley et al. 2005).

LEA uses a fully explicit discretisation scheme. For simplicity the tool makes use of the assumption of an isothermal wall $(\mathrm{Bj}<0.1)$. Another simplification is that only the floor and ceiling are defined as being thermally active. The walls represent thermal resistances only. The thermal energy stored in the structure is equally distributed over floor and ceiling. Their thermal response is defined by the internal convection coefficient. Different to the walls the floor/ ceiling construction is discretised in space. Depending on the defined thermal mass of its construction a number of nodes are defined governing its thermal response in time (Personal communication - Wiebe Zoon; 26 September 2008)

\section{Tool Performance Calibration - Bestest}

To define a starting point for the tool performance analysis both selected tools were bestest-ed. The Bestest procedure allows an inter-software performance evaluation for a number of predefined cases by defining performance limits. The building model used for the tool performance analysis was the Bestest case 600 (Judkoff and Neymark 1995). The case 600 represents a lightweight, one zone building with two south facing windows and $200 \mathrm{~W}$ internal gains. The set points of the ideal plant provide space heating up to $18^{\circ} \mathrm{C}$ and cooling if the room air temperature exceeds $27^{\circ} \mathrm{C}$. The occupation and plant operation period lasts $24 \mathrm{~h}$ on seven days per week. 


\section{Prototyping}

To facilitate the performance comparison, shells were built around the selected tools automating the simulation process. The shell around IES integrates four tools: Simlab, MS Excel, Automate and MS WordPad. The shell around LEA required the integration of Simlab, MS Excel and Matlab. The procedure being applied consisted of the following five stages:

1: Selection of uncertain building specification parameters and identification of standard deviations.

2: Generation of the sample matrix applying Latin hypercube sampling.

3: Simulating the Bestest Case 600 once for each sample.

4: Statistical data analysis, Uncertainty and sensitivity analysis

5. Reporting

The building model is defined differently for the two BPS - tools, what requires different operation regimes for the prototypes. Whilst IES describes a building model by a set of files, LEA only uses one file containing information about model description and operation. This fact resulted in the need to only replace two files containing the sample data prior the simulation, for IES. For LEA each sample to be processed required the generation of a new model description file.

\section{Analysis Criteria}

The foremost assessment criterion when comparing the performance of the two prototypes should be whether the results of the uncertainty and sensitivity analysis lead to the same design decision. Taking account of the comparative nature of the study and the absence of an immediate design problem, two criteria are proposed to evaluate the performance of LEA using IES. The first criterion related to global uncertainties is inspired by the reduction of performance uncertainties during progressing design. It is expected that the performance uncertainties are larger during the early design stages compared to later phases. The second criterion is inspired by the expectation that the design decision finally taken will be the same based on both sets of results.

\section{A: Uncertainty evaluation of performance metrics}

The first criterion is based on the assumption that due to the limited extent of parametric detail available a design proposal during the conceptual design performs less certain than during the detailed design stage. Following the assumption, the LEA prototype performance can be assessed adequate when the global uncertainty of the considered performance metric is equal or larger than calculated by the IES prototype.

B: Impact evaluation of input parameters on sensitivity of performance metric

Global sensitivities are used to assess the impact of concept specification parameters on selected performance metrics. The CDA prototype is expected to perform adequate when the sensitivities calculated in response to specification parameter variations match qualitatively the performance metric sensitivities calculated with the DDA - tool.

\section{SPECIFICATION PARAMETER UNCERTAINTIES}

The tool performance analysis considers material properties only. The first out of two reasons for this limitation is that publications by others, dedicated to the evaluation of the on-site performance of construction materials due to aging, moisture content and temperature changes, are available to extract standard deviations for a multitude of materials (Clarke et al. 1991). The second argument is the possibility to indirectly consider the impact of the modeling abstraction level by aggregating material properties representing building elements as is required for the CDA -tool. Table 1 shows the material properties with their assigned 
standard deviations.

\section{The Sample Matrix}

There are a number of techniques available to conduct a sensitivity analysis such as Monte Carlo + regression analysis -, differential sensitivity (Morris) analysis -, and stochastic sensitivity analysis, among others. The techniques differ in how the sample matrix is generated defining the starting point for the output generation. The Monte Carlo analysis, also referred to as global sensitivity or uncertainty analysis, is characterized by a sample matrix where each of the parameters considered is modified for every sample generated based on a selected sample distribution technique. Latin hypercube sampling was chosen as technique for the sample generation as it gives an improved coverage of the search space.

Table 1: Bestest case 600 - Absolute material properties and assigned standard deviations.

\begin{tabular}{|c|c|c|c|c|c|}
\hline & & Thickness & $\begin{array}{l}\text { Standard } \\
\text { deviation }\end{array}$ & Conductivity & $\begin{array}{c}\text { Standard } \\
\text { deviation }^{[\dagger]}\end{array}$ \\
\hline Units & & (m) & (m) & $(\mathrm{W} / \mathrm{mK})$ & $(\mathrm{W} / \mathrm{mK})$ \\
\hline \multirow[t]{3}{*}{ Wall } & Plasterboard & 0,012 & I & 0,16 & 0,04 \\
\hline & Fiberglas quilt & 0,066 & $0,02^{[\ddagger]}$ & 0,04 & 0,016 \\
\hline & Wood siding & 0,009 & 1 & 0,14 & 0,015 \\
\hline \multirow[t]{2}{*}{ Floor } & Timber flooring & 0,025 & I & 0,14 & 0,0378 \\
\hline & Insulation & 1,003 & I & 0,04 & 0,016 \\
\hline \multirow[t]{3}{*}{ Roof } & Plasterboard & 0,010 & l & 0,16 & 0,04 \\
\hline & Fiberglas quilt & 0,1118 & $0.02^{[\ddagger]}$ & 0,04 & 0,016 \\
\hline & Roof deck & 0,019 & 1 & 0,14 & 0,0238 \\
\hline
\end{tabular}

One question that needs to be addressed before studies of this type is, "How many samples are required to obtain a reasonable accuracy?". MacDonald (2002), and Lomas and Eppel (1992) state that after processing 60-80 samples the improvements in the accuracy of the standard deviation, using the Monte Carlo technique, are marginal. Both publications exclude the number of uncertain parameters to have an impact on the accuracy of the standard deviation of the results. Another source, Simlab 2.2 used as uncertainty analysis processor hints in its help documentation that the minimum number of samples should not be less than 1.5 - times the uncertain parameters considered. In order to comply with both statements and to achieve a good accuracy in the results the number of samples chosen for the analysis was 200 for the conducted 10 parameter uncertainty analysis. That number complies well with other studies reported in literature. A thorough quality check of the resulting sample matrix led to the exclusion of four samples as those contained physically infeasible data points. The conclusions are therefore drawn from results of 196 model simulations.

\section{Parameter aggregation}

Model simplification was included by aggregating input parameter. Parameter aggregation is one technique to reduce the input requirements for CDA - tools. Whilst IES allows the definition of materials using properties such as specific heat capacity (cp), density $(\delta)$ and

\footnotetext{
${ }^{\dagger}$ The standard deviations for the different material and thermo physical properties were calculated based on published data from Clarke et al. (1991),

* or were derived from De Wit (2001).
} 
conductivity (k), LEA only allows the definition of one aggregated parameter $\mathrm{R}_{\mathrm{k}}$ describing the heat conduction through building elements. In order to facilitate an uncertainty analysis, the standard deviations of the individual properties had to be equally aggregated. Table 2 shows the aggregated thermal resistance, $R_{k}$, and corresponding standard deviation. As can be noticed the parameter aggregation results in standard deviations for the wall and roof greater than the mean value of the thermal resistance. This is caused by the fact that the thermal resistance is biased by the uncertainties of the thermal physical material properties.

Table 2: Bestest case 600 - Aggregated material properties and resulting standard deviation.

\begin{tabular}{lll}
\hline & $\begin{array}{c}\text { Thermal Resistance } \\
\left(\mathrm{m}^{2} \mathrm{~K} / \mathrm{W}\right)\end{array}$ & $\begin{array}{c}\text { Standard deviation } \\
\left(\mathrm{m}^{2} \mathrm{~K} / \mathrm{W}\right)\end{array}$ \\
\hline Wall & 1.79 & 2.04 \\
\hline Floor & 25.25 & 21.98 \\
\hline Roof & 2.99 & 3.78 \\
\hline
\end{tabular}

\section{IES and LEA - BESTEST}

The Bestest case 600 was used to calibrate the base line models for the tool performance analysis. Figure 1 indicates the results obtained for both tools, IES VE and LEA. The error bars indicate the Bestest performance limits. The annotated squares and triangles represent tool specific results for annual energy demand for heating and cooling as well as peak heating and cooling loads.

\section{Bestest Case 600 Results Comparision, Lea and IES VE}

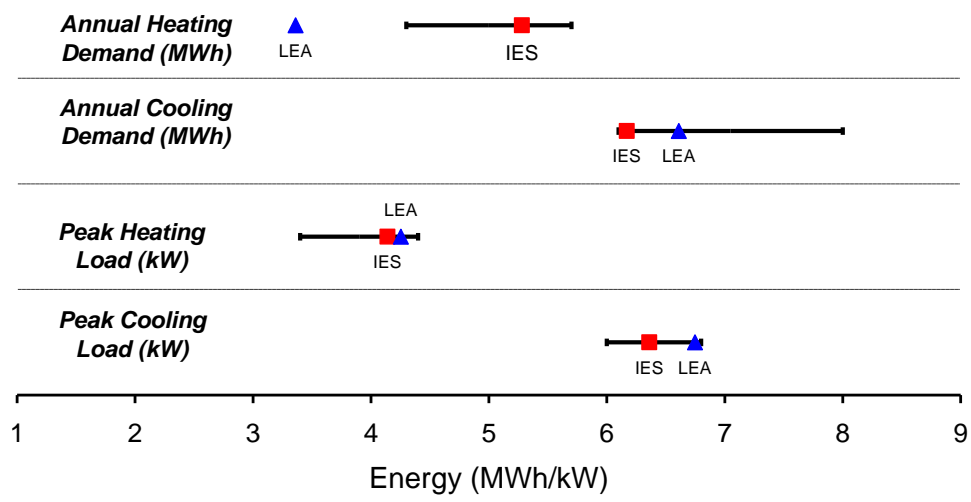

Figure 1: Bestest Case 600 Results, Comparison IES - LEA.

As can be noticed the results for both tools lie well within the limits for the performance metric peak heating and cooling as well as for annual energy demand for cooling. The annual energy demand for heating calculated for LEA stays well below the minimum performance limit. The Bestest procedure suggest to consider qualification cases for compliance checks and in case of disagreements between the result the use of diagnostic cases to isolate its source (Judkoff and Neymark 1995). The consideration of diagnostic cases is beyond the scope of the current study and has not been reported on. Bearing in mind that LEA, a CDA tool, working with abstracted input data it was concluded that complying with three out of four performance criteria is sufficiently accurate for conducting the proposed study. 


\section{RESULTS}

The results presented are structured following the analysis process. Preceding the uncertainty analysis, the results for the Bestest case 600 compliance checks are shown in Appendix A defining a starting point for the uncertainty analysis. The uncertainty analysis itself is structured in two parts. The first part is dedicated to the overall uncertainty and the second part to a comparison of the resulting model sensitivities. The overall uncertainties have been analyzed using the absolute values followed by an analysis of their normalized values for direct comparison of the standard deviations. The performance metrics reported are annual energy demand for heating and cooling as well as the peak heating and cooling loads.

The sensitivities have been considered for each of the four performance metrics individually using the linear partial correlation coefficient (PCC). The PCC allows a sensitivity analysis of linear working systems excluding the effect of correlations between input parameters.

\section{Absolute Results}

Figure 2 and 3 show the absolute range of results calculated across 196 samples. The points at either end of the error bar represent the absolute minimum and maximum values and the annotated squares and circles indicate the corresponding arithmetic mean.

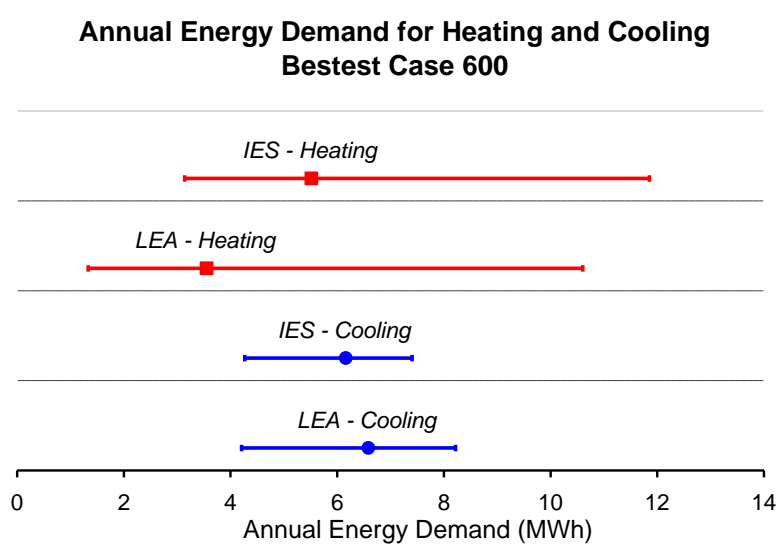

Figure 2: Annual Energy Demand, Absolute Results, Comparison IES - LEA.

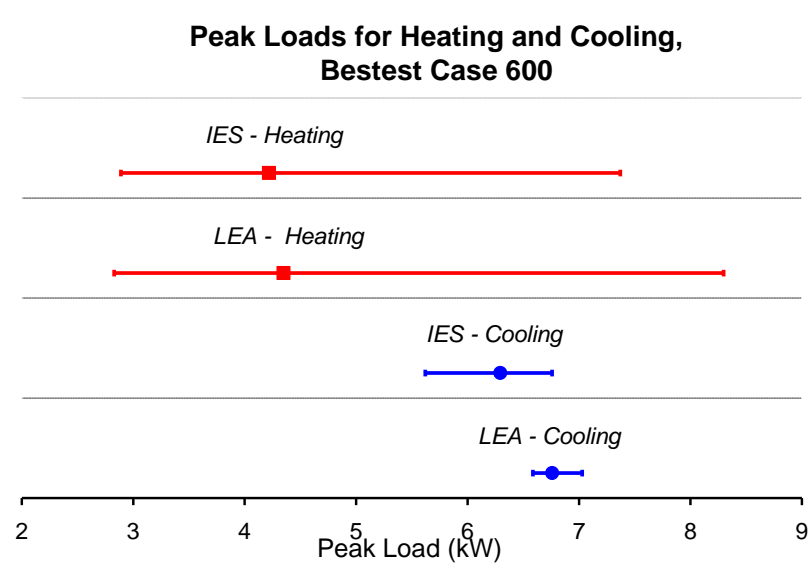

Figure 3: Peak Loads, Absolute Results, Comparison IES - LEA.

As can be noticed the sample coverage between IES and LEA show similarities. However, differences are also noticeable. The entire range of the annual energy demand for heating calculated by LEA is shifted approx. 1MWh to the lower end of the scale compared to the results by IES. Furthermore, the coverage of the peak cooling loads calculated with LEA is significantly smaller than for IES.

\section{Output Uncertainties}

The potential deviation of the performance metric (output uncertainty) resulting from the concept variation specification (input uncertainty) is expected to be of great interest to practitioners. Referring to the Bestest case 600 at hand, practitioners can at first glance recognize the probable occurrence of the four considered performance metrics (see Table 3 ).

Table 3: Standard deviation for absolute results.

\begin{tabular}{cccc}
\hline Annual Heating & Annual Cooling & Peak Heating & Peak Cooling \\
Demand $[\mathrm{MWh}]$ & Demand $[\mathrm{MWh}]$ & Load $[\mathrm{kW}]$ & Load $[\mathrm{kW}]$ \\
\hline
\end{tabular}




\begin{tabular}{lcccccccc}
\cline { 2 - 8 } & Min. & \multicolumn{1}{c}{ Max. } & \multicolumn{1}{c}{ Min. } & Max. & Min. & Max. & Min. & Max. \\
\hline LEA & 2.30 & 4.81 & 5.94 & 7.23 & 3.57 & 5.12 & 6.68 & 6.83 \\
\hline IES & 4.26 & 6.77 & 5.67 & 6.65 & 3.56 & 4.88 & 6.13 & 6.46 \\
\hline
\end{tabular}

To compare and evaluate the resulting uncertainties directly the results were normalized. Table 4 summarizes the normalized standard deviations for the four considered performance metrics. It can be noticed that all but one metric, the peak cooling load, comply with the proposed global uncertainty evaluation criteria. The global parametric output uncertainties calculated with the CDA - tool are larger than the one for the DDA - tool. The standard deviation calculated for the peak cooling load using LEA is less than half the one calculated using IES. The observed disagreement reflects the results presented in the section dedicated to absolute results.

Table 4: Normalized standard deviations for performance metrics.

\begin{tabular}{lrrrr}
\hline & $\begin{array}{c}\text { Annual Heating } \\
\text { Demand }\end{array}$ & \multicolumn{2}{c}{$\begin{array}{c}\text { Annual Cooling } \\
\text { Demand }\end{array}$} & \multicolumn{2}{c}{$\begin{array}{c}\text { Peak Heating } \\
\text { Load }\end{array}$} & \multicolumn{2}{c}{$\begin{array}{c}\text { Peak Cooling } \\
\text { Load }\end{array}$} \\
\hline LEA & 0.353 & 0.099 & 0.178 & $\mathbf{0 . 0 1 1}$ \\
\hline IES & 0.227 & 0.080 & 0.156 & 0.026 \\
\hline
\end{tabular}

Note: The highlighted parameter indicates non-compliance with the global uncertainty evaluation criteria.

\section{Model Sensitivity Comparison}

Once the global uncertainties of the performance metrics have been established the interest of practitioners should focus on their minimization. To initiate the minimization exercise, it becomes necessary to differentiate between sensitive and insensitive input parameters. The sensitive parameters have a greater relevance towards influencing the considered performance metric than insensitive parameters.

Based on finding in (Lomas and Eppel 1992), the linear partial correlation coefficient (PCC) has been chosen as indicator for the systems parameter influence on the performance metrics. The PCC was selected as it was assumed that the system consisting of material property uncertainties and simulation model works linear. Furthermore, the PCC enables identifying the correlation of the input parameter and the output parameter, cleaned of effect caused by inter-correlations between input parameters (Saltelli et al. 2006).

Figures 4-7 visualize a comparison of PCC's derived from the uncertainty based global sensitivity analysis using SimLab 2.2. The graphs show the sensitivities of four performance metrics to the uncertainty of ten input parameters. Whilst a large PCC indicates a high sensitivity, a small PCC indicates insensitivity. The bars have been ordered following the ranking of the CDA - tool. The top bar identifies the most sensitive and relevant input parameter the bottom bar shows the least sensitive and irrelevant parameter. The algebraic sign of the PCC indicates the parameter impact, which is positive or negative. The PCC's calculated with the DDA - tool have been arranged using the parameter ranking of the CDA tool. The resulting ranking is therefore not strictly descending but enables a direct comparison of the parameter specific PCC between the tools. 
Annual Heating Demand, PCC based Sensitivites

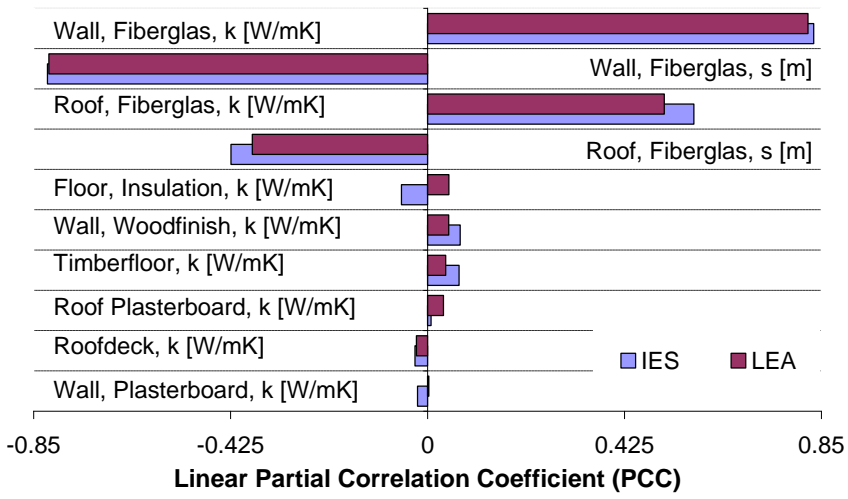

Figure 4: Annual Energy Demand for Heating, PCC based Sensitivities, Comparison IES - LEA.
Annual Cooling Demand, PCC based Sensitivites

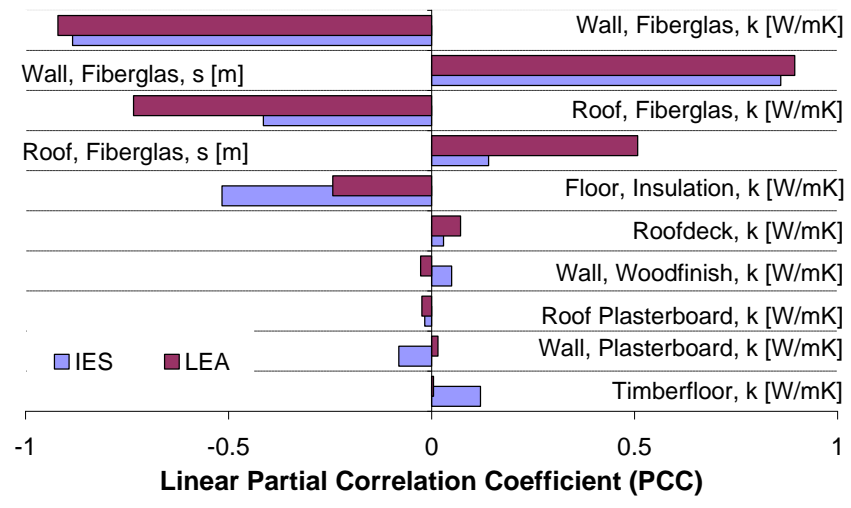

Figure 5: Annual Energy Demand for Cooling, PCC based Sensitivities, Comparison IES - LEA.

\section{Annual Energy Demand for Heating (ADH)}

Figure 4 shows a good visual agreement between LEA and IES. The most relevant parameter can be clearly identified as being the conductivity of the wall insulation followed by the thickness of the wall insulation. The third sensitive parameter is the conductivity of the roof insulation and the fourth sensitive parameter being the thickness of the roof insulation (see table A1). The remaining six parameters do seem to have a negligible small individual impact on the uncertainty of the $\mathrm{ADH}$. The sensitivity ranking of the two tools is in good agreement. Another fact that can be noticed is that parameters have opposing influences for the two tools. For example, the conductivities of the floor insulation and wall plasterboard show a negative PCC for IES and a positive PCC for LEA. The phenomenon is refereed to as "Reversed sensitivities", and is elaborated on in Appendix C.

\section{Annual Energy Demand for Cooling (ADC)}

Figure 5, showing the PCC based sensitivities for the ADC gives a less ordered picture than has been observed for ADH. For both tools five relevant parameters can be identified. The parameter sensitivity ranking for the five parameters is shown in table A2. Deviant to LEA, IES shows a different ranking. Whilst LEA results rank the conductivity of the floor insulation the fifth most sensitive parameter, IES ranks the same parameter third. The remaining two parameters are devalued one rank but keep their order. Reversed sensitivities can be noticed for the conductivities of wall wood finish and wall plasterboard.

The parameters that stand out as having an impact on the ranking describe the thermal behavior of the floor construction. The floor construction defined for the Bestest case 600 was set to decouple the space from the ground. Due to that reason the annual cooling demand and peak load react exceptionally sensitive to parametric changes.

\section{Peak Heating Load (PHL)}

Figure 6 shows a good visual agreement between LEA and IES. The most relevant parameter can be clearly identified as being the conductivity of the wall insulation followed by the thickness of the wall insulation. The third sensitive parameter is the conductivity of the roof insulation and the fourth sensitive parameter being the thickness of the roof insulation (see table A3). The remaining six parameters do seem to have a negligible small individual impact on the uncertainty of the peak heating load. The sensitivity ranking of the two tools is in good agreement. Reversed sensitivities do not occur.

\section{Peak Cooling Load (PCL)}


Figure 7, showing the PCC based sensitivities for the PCL shows reduced similarities between the two tools compared to ADC. For both tools five relevant parameters can be identified. However, whilst previously the top five ranks were occupied by the same parameters in changing order, different parameters occupy the top five ranks for the PCL. The parameter sensitivity ranking for the five relevant parameters is shown in table A4. Deviant to LEA, IES shows a different ranking. Whilst both show the same ranking for the same two most relevant parameters the following ranks are occupied differently. Interesting is that IES ranks the timber floor conductivity seventh, whilst LEA results rank the parameter fourth. Furthermore, it surprises that IES ranks the roof insulation thickness fifth, whilst LEA ranks it ninth. Reversed sensitivities can be noticed for the conductivities of the wall plasterboard and roof plasterboard.

Peak Heating Load, PCC based Sensitivites

\begin{tabular}{l|c} 
Wall, Fiberglas, $\mathrm{k}[\mathrm{W} / \mathrm{mK}]$ \\
\hline Roof, Fiberglas, $\mathrm{k}[\mathrm{W} / \mathrm{mK}]$ \\
\hline
\end{tabular}

Figure 6: Peak Heating Load,

PCC based Sensitivities, Comparison between IES - LEA.
Peak Cooling Load, PCC based Sensitivites

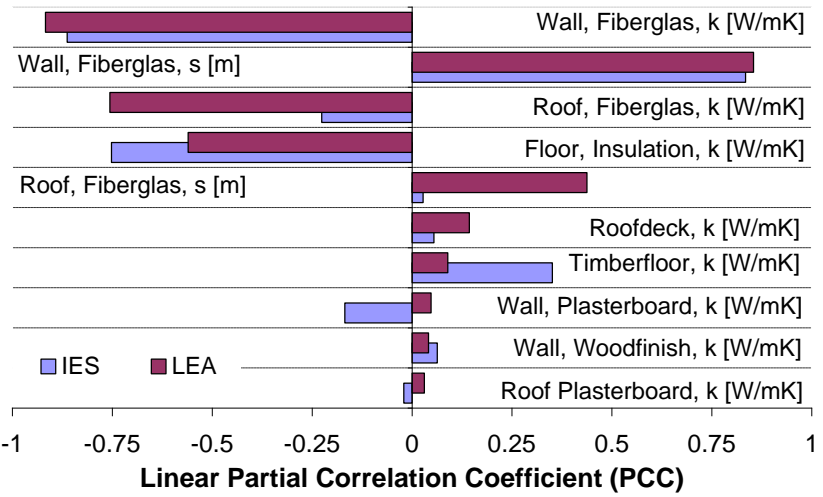

Figure 7: Peak Cooling Load, PCC based Sensitivities, Comparison between IES - LEA.

\section{Discussion}

The conceptual design stage has some unique characteristics such as many unknown parameters and a large number of considered design options. Due to those characteristics there is a limited need for precise simulation results. However, the tools should facilitate a quick turn around of performance evaluations of the design options. Subsequent, and different, to detailed design tools, conceptual design tools need to be fast-running and very user-friendly facilitating the tool use for a multidisciplinary user group with different levels of background in building science.

In order to facilitate the evaluation of the two prototypes, the input parameters for the Detailed Design Analysis (DDA) tool had to be aggregated to be used as input to the Conceptual Design Analysis (CDA) tool. Parameter aggregation made it possible to consider input parameter uncertainties that cannot be defined directly with the CDA tool's interface.

Two criteria are introduced to evaluate the prototypes. The criteria propose to assess the CDA tool performance adequate, if its results support the same design decision as the DDA tool.

The performance of the CDA tool complies with the first proposed criterion if the global uncertainties are equal or larger than the DDA. It is inspired by the performance uncertainty reduction observed during the design process (Struck et al. 2008). The criterion is not fulfilled. Not all performance metrics show equal or larger global uncertainties for the CDA tool compared to the DDA tool. Using the normalized standard deviation as indicator for uncertainty, the CDA tool shows less than half the uncertainty of the DDA tool for the peak cooling load.

As a prerequisite to arrive at the same design decision the tools are required to rank the impact of the input parameters on the performance metrics similarly high and strong, as formulated in the second criterion. The degree of agreement of sensitivities for both tools is 
different for heating and cooling related performance metrics.

The heating related performance metrics show a good agreement for high and low ranking input parameters. Furthermore, there is a clear difference noticeable between four high and six low ranking parameters. The results for heating related performance metrics comply well with the posed criterion.

The results from the sensitivity analysis for peak heating load and annual heating demand did show a good agreement with the sensitivities obtained with the DDA tool. It can be noticed that the same four parameters have a strong impact on the output range. The most important input parameters, in descending order, are: conductivity of the wall insulation material, thickness of the wall insulation material, conductivity of the roof insulation material and thickness of the roof insulation material. The remaining six parameters have a negligible small impact on the global output sensitivity.

The degree of agreement between the tools is reduced significantly for the performance metrics annual energy demand for cooling and peak cooling load. Only the two most sensitive parameters show a good agreement for annual cooling demand and peak cooling load. The two most important parameters are: conductivity of the wall insulation material and thickness of the wall insulation material. It can be noticed that LEA differently to IES ranks the importance of the roof insulation much higher. Furthermore, the impact of the input parameters reduces gradually from the most sensitive to the least sensitive parameter.

The varying impact of the input parameters on the uncertainty of the performance metric can be attributed to the tool specific representation of the thermal response of the building structure as it is limited to cooling related performance metrics. A deeper analysis of the underlying causes cannot be conducted as both tools represent commercial tool for which the access to the calculation engine is restricted.

It is important to know about the limits of the underlying physical model when using parameter aggregation. Although both models use the walls thermal resistance to assess the static and dynamic heat loss the model representing the structures thermal response is different leading to differences in the impact of input parameters on the heat gains.

The results show that the assessment criteria are not met for all performance metrics. That causes questioning the criteria's applicability to evaluate the tools and the limited extend of the study.

One reason why the criteria are insufficient for evaluating the prototypes performance is the possibility that the approximation adopted in the LEA model, in some cases, reduces the uncertainty.

Another reason for the criteria failing to lead to direct conclusions is the limited selection of input parameters. The focus on establishing and comparing uncertainties and sensitivities for material properties only does not necessarily reflect the needs during the conceptual design stage and thereby does not correspond to the expected use of the tool.

There was no indication in either uncertainties nor BESTEST results that the sensitivities for the cooling related performance metrics would be less comparable than heating related performance metrics.

\section{CONCLUSION}

This article proposes to enhance the use of performance simulation in concept design by enabling uncertainty and sensitivity analysis focusing on estimating performance uncertainties. The first logical step was to equip a tool specifically developed to support the conceptual design stage with capabilities to conduct sensitivity and uncertainty analysis and to compare its performance to a detailed design analysis tool.

In this study the performance of two prototypes dedicated to facilitate an uncertainty and global sensitivity analysis are compared. The first prototype is build around one concept design analysis tool, LEA. The second prototype is based on one detailed design analysis tool, IES. The aim is to identify if the tools compute comparable results and subsequently if conceptual design analysis tools provide the same quality of results to support design decision 
as detailed design analysis tools.

The feasibility of extending conceptual and detailed design tools with capabilities to conduct uncertainty and sensitivity analysis is demonstrated.

Uncertainties were introduced by assigning standard deviations to ten input parameters representing material properties. To assess the results, output uncertainties and global sensitivities were considered. Two criteria were defined against which the results were evaluated.

Preceding the uncertainty and global sensitivity analysis both tools were Bestest-ed using the first Bestest case 600. It was found that IES complies with all four performance limits, which are annual demand for heating and cooling as well as peak heating and cooling loads. Performing the test with LEA, it was found that three out of four performance limits could be met. The annual heating demand does not comply with the set limits. However, considering the tool's abstraction level it was decided to proceed with the comparative study.

Concluding the analysis it was found that the not Bestest compliant performance metric, annual heating demand, shows no irregularities relating to the quality of results from uncertainty and sensitivity analysis. On the contrary, the heating related performance metrics comply well with the posed criteria.

To enable the tool evaluation input parameters for the simplified tool had to be aggregated. It was found that the case specific input parameter aggregation did not hinder conducting an analysis of their impact on the output parameter, sensitivity analysis. As IES complies with the Bestest it results are qualitatively better than those from LEA. To evaluate the results of LEA prototype the IES results were used as benchmark.

The differences noticed in uncertainties and sensitivities between simplified and detailed tools can be attributed to the limited detail of the model underlying the simplified tool. However, no detailed analysis and comparison of the tool physical models was possible as the tools are commercial tools with their models only briefly described in literature (see section Tool description).

It was found that in one instance, for the peak cooling load, the calculated uncertainty was smaller for the simplified analysis tool than observed for the detailed analysis tool. The parameter sensitivities do agree well for the heating related performance metrics but very little for cooling related performance metrics. The differences in results lead to the conclusion that none of the two proposed tool evaluation criteria is sufficient. That means that it cannot be stated with confidence that the design decision taken based on results from the simplified tools will be the same as taken based on the detailed design tool.

Consequently, it can be stated that the type of adaptation chosen to facilitate an early tool use, e.g., detailed tools with simplified interfaces versus abstracted models with corresponding interfaces, does have an influence on the quality of the results when used for uncertainty and sensitivity analysis. The results indicate that, when using uncertainty and sensitivity analysis to enhance early tool use, detailed tools with simplified interfaces pose the most promising way forward.

Uncertainty and sensitivity analysis shows potential for tool developers to support model diagnostics. Big differences in sensitivities as well as reversed sensitivities might be used as indicators towards specific model differences, e.g., the representation of the thermal storage capacity.

As is the case with commercial software tools limited knowledge is available about the underlying physical models. Therefore, it is difficult to inter-compare uncertainties for predicted performance metrics. It is generally accepted to expect the performance of a building proposal to be more uncertain during conceptual design than for detailed design. Still, that assumption does not hold for comparing uncertainties predicted with CDA tools and DDA tools by using the same input uncertainties.

\section{FUTURE WORK}

The uncertainties considered were limited to physical uncertainties (uncertainties related to material properties). Traditionally, those uncertainties are considered to calibrate virtual 
models, which is not the primary need for using BPS tools in conceptual design. Design uncertainties, such as wall to window area ratios or space surface area to volume ratios represent much better typical conceptual design questions. Therefore, future work will include the consideration of uncertainties related to typical conceptual design questions.

It cannot be excluded that the model underlying the simplified tool leads to the variation of sensitivities. Therefore, future work will be dedicated using detailed tools with state of the art and detailed calculation models.

Inaccuracies of models can go either way over or underestimating the error. So expecting the uncertainty to be bigger or smaller is more realistic than simply stating, the uncertainty is expected to be bigger. More work is required to establish performance criteria to quantitatively evaluate the BPS tools with uncertainty and sensitivity analysis capabilities.

\section{REFERENCES}

Clarke, J. A., Yaneske, P. P., and Pinney, A. A. (1991). "The harmonisation of thermal properties of building materials." Report TN91/6, BRE, Garston, Watford, UK.

Crawley, D. B., Hand, J. W., Kummert, M., and Griffith, B. T. (2005). "Contrasting the capabilities of building energy performance simulation programs."

Cropp, R. A., and Braddock, R. D. (2002). "The New Morris Method: an efficient secondorder screening method." Reliability Engineering and System Safety, 78, 77-83.

Hopfe, C. J., Struck, C., G., U. H., and Hensen, J. "Computational tools for building services design - professional's practice and wishes." 17th International Air-conditioning and Ventilation Conference

Prague, p. 5, on CD.

Hopfe, C. J., Struck, C., Ulukavak Harputlugil, G., Hensen, J., and Wilde, P. d. "Exploration of using building performance simulation tools for conceptual building design." IBPSANVL conference, Delft, Netherlands, 8 pages on CD.

Judkoff, R., and Neymark, J. (1995). "International energy agency building energy simulation test (BESTEST) and diagnostic method." NREL/TP-472-6231, National Renewable Energy Laboratory.

Lomas, K. J., and Eppel, H. (1992). "Sensitivity analysis techniques for building thermal simulation programs." Energy and Buildings, 19(1), 21-44.

Macdonald, I. (2002). "Quantifying the effects of uncertainty in building simulation," University of Strathclyde, Glasgow, UK.

Saltelli, A., Ratto, M., Tarantola, S., and Campolongo, F. (2006). "Sensitivity analysis practices: Strategies for model-based inference." Reliability Engineering \& System Safety, 91(10-11), 1109-1125.

Struck, C., de Wilde, P., Hopfe, C. J., and Hensen, J. "An Exploration of the Option Space in Student Design Projects for Uncertainty and Sensitivity Analysis with Performance Simulation." Intelligent Computing in Engineering (ICE08) - A Joint US-European Workshop, Plymouth, UK, pp.9-on CD.

Struck, C., and Hensen, J. "Uncertainty analysis for conceptual building design - A review of input data." 1st Int. IBPSA Germany/Austria Conf. BauSIM, Technische Universitaet Muenchen, 4 pages on CD.

Urban, B., and Glicksman, L. "The MIT Design Advisor - a fast, simple tool for energy efficient building design." SimBuild 2006, Massachusetts Institute of Technology, Cambridge, MA, USA.

Vabi, B. (2006). User Manual h.e.n.k. v3.0 - Handleiding h.e.n.k. v3.0, Vabi BV., Delft, Netherlands.

Verbeeck, G., and Hens, H. (2007). "Life Cycle Optimization of Extremely Low Energy Dwellings." Journal of Building Physics, 31(2), 143-177. 
Wit, M. S. d. (2001). Uncertainty in predictions of thermal comfort in buildings, Technische Universiteit Delft, Delft, The Netherlands.

\section{APPENDIX A - GLOBAL SENSITIVITY PARAMETER RANKING}

\section{Annual Energy Demand For Heating (ADH) \& Cooling (ADC)}

Table A1: ADH Top 4, Most sensitive parameters, Ranking comparison.

\begin{tabular}{ccc}
\hline & \multicolumn{2}{c}{ Ranking } \\
\cline { 2 - 3 } Input parameter & LEA & IES \\
Wall, Fiberglas, $\mathrm{k}$ & 1 & 1 \\
\hline Wall, Fiberglas, s & 2 & 2 \\
\hline Roof, Fiberglas, $\mathrm{k}$ & 3 & 3 \\
\hline Roof, Fiberglas, s & 4 & 4 \\
\hline
\end{tabular}

Table A2: ADC Top 5, Most sensitive parameters, Ranking comparison

\begin{tabular}{lcc}
\hline & \multicolumn{2}{c}{ Ranking } \\
\cline { 2 - 3 } \multicolumn{1}{c}{ Input parameter } & LEA & IES \\
\hline Wall, Fiberglas, $\mathrm{k}$ & 1 & 1 \\
\hline Wall, Fiberglas, $\mathrm{s}$ & 2 & 2 \\
\hline Roof, Fiberglas, $\mathrm{k}$ & 3 & 4 \\
\hline Roof, Fiberglas, $\mathrm{s}$ & 4 & 5 \\
\hline Floor, Insulation, $\mathrm{k}$ & 5 & 3 \\
\hline
\end{tabular}

\section{Peak Heating (PHL) \& Cooling Load (PCL)}

Table A3: PHL Top 4, Most sensitive parameters, Ranking comparison.

\begin{tabular}{ccc}
\hline & \multicolumn{2}{c}{ Ranking } \\
\cline { 2 - 3 } Input parameter & LEA & IES \\
\hline Wall, Fiberglas, $\mathrm{k}$ & 1 & 1 \\
\hline Wall, Fiberglas, $\mathrm{s}$ & 2 & 2 \\
\hline Roof, Fiberglas, $\mathrm{k}$ & 3 & 3 \\
\hline Roof, Fiberglas, $\mathrm{s}$ & 4 & 4 \\
\hline
\end{tabular}

Table A4: PCL Top 5, Most sensitive parameters, Ranking comparison.

\begin{tabular}{lcc}
\hline \multirow{2}{*}{ Input parameter } & \multicolumn{2}{c}{ Ranking } \\
\cline { 2 - 3 } & LEA & IES \\
\hline Wall, Fiberglas, $\mathrm{k}$ & 1 & 1 \\
\hline Wall, Fiberglas, $\mathrm{s}$ & 2 & 2 \\
\hline Floor, Insulation, $\mathrm{k}$ & 3 & 4 \\
\hline Timber floor, $\mathrm{k}$ & 4 & 7 \\
\hline
\end{tabular}

\section{APPENDIX B - SENSITIVITY INCONSISTENCY}

The algebraic sign (plus/minus) in front of the PCC indicates the direction of the parameter specific impact. For example, an increase of the wall insulation conductivity increases the annual heating demand and an increase of the wall insulation thickness reduces the annual heating demand. It was expected that the PCC's for both tools for the same parameter show the same algebraic sign to indicate the direction of impact. However, a number of input parameters show opposing algebraic signs in front of the PCC. Table A6 identifies the parameter showing sensitivity inconsistencies between LEA and IES of concern.

Table A5: List of parameters showing reversed sensitivities between IEA and LEA.

\begin{tabular}{|c|c|c|c|}
\hline Heating & & oling & \\
\hline Annual demand & Peak load & Annual demand & Peak load \\
\hline
\end{tabular}




\begin{tabular}{llll}
\hline Wall, Plasterboard, k & $/$ & Wall, Plasterboard, k & Wall, Plasterboard, k \\
\hline Floor, Insulation, k & $/$ & Wall, Wood finish, k & Roof Plasterboard, k \\
\hline
\end{tabular}

The most important parameters are defined by PCC's with the same algebraic sign for each of the performance metrics. At the same time the algebraic sign occurs self-explanatory reversed, when comparing input parameter specific PPC's for annual heating and cooling as well as peak heating and cooling. Following the above a consistency check was conducted for the parameters identified with reversed sensitivities to identify the tool specific behavior of the parameter. Table A6, identifies the parameter and associated tool computing a unidirectional impact of PCC on annual heating and cooling demand as well as peak heat and cooling load.

Table A6: Sensitivity inconsistencies, Parameters resulting in a uni-directional impact across annual demand/ peak loads.

\begin{tabular}{lll}
\hline & IES & LEA \\
\hline Annual demand & Wall, Plasterboard, $\mathrm{k}$ & Wall, Plasterboard, $\mathrm{k}$ \\
\hline & Wall, Wood finish, $\mathrm{k}$ \\
\hline & Floor, Insulation, $\mathrm{k}$ \\
\hline Peak load & Wall, Plasterboard, $\mathrm{k}$ & Roof Plasterboard, $\mathrm{k}$ \\
\hline
\end{tabular}

It can be noticed that the unidirectional impact only occurs for parameters describing the heat transfer for internal or externally exposed layers. So an explanation could be sought in the methodology the tools of representing the surface heat transfer, subsequently acting on energy demand and peak loads. 